\title{
Influencia del cruce industrial en los rendimientos productivos y la calidad de la canal de corderos de la raza ovina Segureña
}

\author{
Blasco, M. ${ }^{\circledR} ;$ Campo, M.M. ${ }^{1}$; Balado, J. ${ }^{2}$ y Sañudo, C. ${ }^{1}$
}

\begin{abstract}
'Departamento de Producción Animal y Ciencia de los Alimentos. Universidad de Zaragoza. Zaragoza. España.
\end{abstract} 2Diputación de Castellón. Ares del Maestre. Castellón. España.

PALABRAS CLAVE ADICIONALES

Ovino.

Texel.

Cruzamiento.

Crecimiento.

Composición tisular.

\section{RESUMEN}

Se han estudiado los rendimientos productivos (peso al nacimiento, peso al sacrificio, y ganancia media diaria), así como los parámetros de calidad de la canal (conformación, engrasamiento, índices de compacidad de la canal y de la pierna, y composición tisular) de corderos provenientes de la raza Segureña y del cruce industrial de ésta con la raza Texel. Se han observado mejoras en los animales cruzados, tanto en la productividad: mayor peso al nacimiento y al sacrificio y una mayor ganancia media diaria de peso, como en la calidad de la canal: mejor conformación, menor engrasamiento, y una mayor composición muscular en contraposición a un menor porcentaje de grasa. Los resultados obtenidos indican que el cruce entre estas dos razas podría ser una herramienta recomendable a desarrollar y utilizar en determinadas explotaciones y especificaciones de producción.

Effect of crossbreeding on productive yield and carcass quality in Segureña lambs

\section{SUMMARY}

Productive traits (birth weight, slaughter weight, average daily gain), carcass quality traits (conformation, fatness score, carcass and leg blockness and tissue composition) in lambs from either pure Segureña breed or crossbred with Texel were studied. Improvements in the crossbred animals were observed, both in productive traits: higher birth and slaughter weights and average daily gain, and in carcass quality: bigger conformation, lower fatness score and a higher muscle composition in contrast with a lower fat percentage. The results indicate that the crossbreeding between these two breeds could be a recommended tool to develop and use in certain farms and production conditions.

\section{INFORMACIÓN}

Cronología del artículo.

Recibido/received: 21.03 .15

Aceptado/Accept: 14.03.16

Online: 15.09.16

Correspondencia a los autores/Contact e-mail:

jblascos@unizar.es

\section{INTRODUCCIÓN}

Diversos factores, políticos, técnicos, sociales y económicos han puesto al Sector Ovino en situación de crisis en muchas zonas de Europa. Por ello, se deben buscar nuevas alternativas o reinventar las ya existentes, haciendo frente a un mercado en el que la meta debe ser la obtención de un producto de calidad. El cruce industrial, entendido como el acoplamiento de machos de razas cárnicas con hembras de razas lecheras o locales, fue una técnica muy utilizada en España entre los años 70 y 90 del siglo XX. Su principal objetivo es mejorar los índices productivos y la calidad de la canal de las razas locales, posibilitando unos mayores pesos al sacrificio (Nitter, 1974; Kempster et al., 1987; Osorio et al., 1998; Kremer et al., 2004). En este sentido, se han desarrollado numerosos trabajos de cruzamiento, especialmente con las razas llamadas Ovinos Precoces (Espejo et al., 1977; Esteban, 2003) y otras (Valls, 1980; Sierra, 1981). Hoy en día, en amplias zonas, el uso del cruce industrial ha decaído notablemente, y posiblemente más de lo técnicamente justificable. El precio de los sementales, la mayor complejidad del manejo del rebaño al tener que mantener las razas de los progenitores en pureza o la compra 
Tabla I. Efecto del genotipo (G: Puro= Segureña x Segureña; Texel x= Texel x Segureña), sexo (S: M= macho ; H= hembra) y tipo de parto (P) sobre parámetros productivos (media \pm desviación estándar) (Effect of genotype (G: Pure= Segureña $x$ Segureña; Texel $x=$ Texel $x$ Segureña), $\operatorname{sex}(S: M=$ male; $H=$ female) and birth $(P)$ in productive traits (mean \pm standard deviation).

\begin{tabular}{|c|c|c|c|c|c|c|c|c|c|}
\hline & \multicolumn{2}{|c|}{ Genotipo } & \multicolumn{2}{|c|}{ Sexo } & \multicolumn{2}{|c|}{ Parto } & \multirow[t]{2}{*}{$\mathrm{G}^{*}$} & \multirow[t]{2}{*}{$\mathrm{S}^{*}$} & \multirow[t]{2}{*}{$P^{*}$} \\
\hline & $\begin{array}{c}\text { Puro } \\
n=68\end{array}$ & $\begin{array}{c}\text { Texel x } \\
n=68\end{array}$ & $\begin{array}{c}\text { M } \\
n=72\end{array}$ & $\begin{array}{c}\mathrm{H} \\
n=64\end{array}$ & $\begin{array}{l}\text { Simple } \\
n=68\end{array}$ & $\begin{array}{c}\text { Doble } \\
n=68\end{array}$ & & & \\
\hline (kg) & $4,1 \pm 0,7$ & $5,1 \pm 1,1$ & $4,8 \pm 1,0$ & $4,5 \pm 1,0$ & $5,3 \pm 1,2$ & $4,3 \pm 0,8$ & 0,000 & 0,323 & 0,000 \\
\hline eso sacrificio (kg) & $21,8 \pm 4,2$ & $24,9 \pm 5,6$ & $24,7 \pm 5,1$ & $21,9 \pm 5,0$ & $26,5 \pm 5,3$ & $22,1 \pm 4,6$ & 0,000 & 0,035 & 0,000 \\
\hline Edad sacrificio (días) & $72,4 \pm 5,9$ & $72,1 \pm 5,4$ & $73,0 \pm 5,2$ & $71,5 \pm 6,0$ & $72,4 \pm 5,8$ & $72,2 \pm 5,6$ & 0,375 & 0,439 & 0,821 \\
\hline GMD (g) & $243,0 \pm 45,9$ & $271,9 \pm 60,0$ & $271,3 \pm 55,9$ & $241,8 \pm 50,3$ & $290,7 \pm 57,1$ & $244,1 \pm 48,5$ & 0,002 & 0,002 & 0,000 \\
\hline
\end{tabular}

GMD= Ganancia media diaria desde el nacimiento hasta el sacrificio; *Valores de $p$.

de fuera de la explotación de los mismos y la implantación de marcas de calidad, con un valor económico añadido basado en lo autóctono, en la mayor parte de los casos en pureza, han sido unas de las principales causas de esa caída. La raza Segureña, del tronco entrefino, está ampliamente extendida por España, incluyendo zonas con una media o baja vocación ovina (Esteban, 2003) como la Comunidad Valenciana o Andalucía Occidental. El cruce con la raza Texel, raza muy antigua de origen holandés que presenta canales magras y de gran morfología cárnica (Sañudo, 2011), podría ser muy útil en zonas en donde la grasa sea claramente penalizada y el peso de canal elevado un factor positivo de comercialización (exportación, venta de determinados despieces y presentaciones)

Por todo ello en este trabajo se han estudiado determinados índices productivos y de calidad de canal en animales puros de raza Segureña y cruzados con machos de raza Texel.

\section{MATERIAL Y MÉTODOS}

- Datos productivos. Se han utilizado dos grupos de hembras homogéneos seleccionadas al azar de raza Segureña en pureza, del rebaño de la Diputación Provincial de Castellón (España). Uno de ellos se cubrió con cinco machos Texel y el otro con cinco machos de raza Segureña. El periodo de cubriciones duró un periodo de 51 días. Los nacimientos se produjeron a partir del 20 de marzo de 2014, controlándose el tipo de parto, el peso al nacimiento, y cada 15 días hasta el sacrificio su peso vivo individual. Los corderos se alimentaron con la madre y, tras el destete, a los 45 días de edad, con pienso comercial (cordestar cebo ligero - NANTA®) y paja de cereal ad libitum. Con estos datos, se calcularon diversos índices productivos (tabla I).

- Rendimientos y calidad de la canal. Del total de animales nacidos se seleccionaron 10 machos de edad y pesos similares en el momento del sacrificio. Sobre ellos se va-

Tabla II. Efecto del genotipo sobre la calidad de la canal de corderos puros y cruzados de raza Texel x Segureña (media \pm desviación estándar) (Effect of genotype on carcass quality in pure and crossbred Texel $x$ Segureña lambs (mean \pm standard deviation).

\begin{tabular}{|c|c|c|c|}
\hline & \multicolumn{2}{|c|}{ Genotipo } & \multirow{2}{*}{$\mathrm{p}$} \\
\hline & Segureño & Texel $x$ & \\
\hline Rendimiento y calidad de la canal & $\mathrm{n}=10$ & $\mathrm{n}=10$ & \\
\hline $\mathrm{PCC}(\mathrm{kg})$ & $11,3 \pm 0,6$ & $11,0 \pm 0,6$ & 0,230 \\
\hline Rendimiento canal (\%) & $45,4 \pm 1,5$ & $45,3 \pm 1,6$ & 0,865 \\
\hline Conformación ${ }^{1}$ & $2,8 \pm 0,5$ & $3,5 \pm 0,5$ & 0,010 \\
\hline Engrasamiento $^{2}$ & $3,4 \pm 0,3$ & $2,2 \pm 0,5$ & 0,000 \\
\hline Compacidad de la canal $(\mathrm{kg} / \mathrm{cm})$ & $0,22 \pm 0,01$ & $0,23 \pm 0,01$ & 0,130 \\
\hline Compacidad de la pierna $(\mathrm{kg} / \mathrm{cm})$ & $0,66 \pm 0,02$ & $0,71 \pm 0,03$ & 0,002 \\
\hline Composición tisular espalda (\%) & $\mathrm{n}=10$ & $\mathrm{n}=10$ & \\
\hline Músculo & $61,5 \pm 1,8$ & $66,0 \pm 1,8$ & 0,000 \\
\hline Grasa preescapular & $2,5 \pm 0,3$ & $1,6 \pm 0,3$ & 0,000 \\
\hline Grasa subcutánea & $8,3 \pm 1,3$ & $4,9 \pm 1,2$ & 0,000 \\
\hline Grasa intermuscular & $6,1 \pm 1,1$ & $5,3 \pm 0,9$ & 0,076 \\
\hline Grasa total & $16,9 \pm 2,0$ & $11,8 \pm 1,5$ & 0,000 \\
\hline Hueso & $20,5 \pm 0,8$ & $21,2 \pm 0,9$ & 0,078 \\
\hline Otros & $1,1 \pm 0,3$ & $0,9 \pm 0,3$ & 0,274 \\
\hline
\end{tabular}

${ }^{1}$ Escala 1, muy mala-5, muy buena; ${ }^{2}$ Escala 1 , poco graso-5 muy graso. 
loró su peso canal, conformación y engrasamiento según una escala de 5 puntos (Colomer et al., 1988), y se calcularon los índices de compacidad de la canal y de la pierna y la composición tisular de la espalda tras el despiece normalizado (Vergara, 2005) (tabla II).

- Estudio estadístico. Las variables productivas del rebaño completo se han analizado con el paquete estadístico SPSS (19.0) a través de un modelo lineal general con los efectos genotipo (Segureña $x$ Segureña y Texel $x$ Segureña), sexo (macho y hembra) y tipo de parto (simple o doble, considerando el único parto triple habido como doble) y sus interacciones, las cuales no han sido significativas en las variables estudiadas. Las variables de los 20 animales utilizados en el estudio de canal se han analizado con el efecto genotipo como único efecto fijo.

\section{RESULTADOS Y DISCUSIÓN}

En la tabla I se observa un efecto significativo $(\mathrm{p} \leq 0,001)$, y similar en magnitud, del genotipo y del tipo de parto sobre las variables productivas analizadas, excepto, como era de esperar al haber sido seleccionados los animales por este criterio, en la edad de sacrificio. En el peso al nacimiento, los animales cruzados con Texel, raza más cárnica y de mayor peso adulto, presentaron de media un kilo más de peso que aquellos de raza Segureña. Esta influencia del genotipo sobre el peso vivo se arrastra hasta el sacrificio ya que, a la misma edad, los animales cruzados mostraron $3 \mathrm{~kg}$ más de peso vivo que los puros, debido, además, a su mayor ganancia media diaria (GMD), $29 \mathrm{~g}$ superior que la de los animales puros. Estos resultados son coincidentes con los obtenidos por Cardoso et al. (2013) en el cruce de Texel con la raza local brasileña Santa Inés y Mújica et al. (2005) en el cruce de Texel con la raza Corriedale, si bien otros autores que han estudiado el mismo cruce con Santa Inés no han encontrado tal mejoría (Da Silva et al., 2014). El sexo fue también significativo para el peso al sacrificio $(\mathrm{p} \leq 0,05)$ y la GMD ( $\mathrm{p} \leq 0,01)$, mostrando los machos un mayor peso y una mayor GMD. Las diferencias debidas al tipo de parto implican un mayor peso al nacimiento de aquellos animales procedentes de parto simple, siendo estas diferencias mayores al sacrificio debidas a la mayor GMD de los animales procedentes de parto simple, fundamentalmente debidas a un mayor crecimiento durante el periodo de lactancia, al no tener competencia en el aporte de leche de la madre con respecto a aquellos animales procedentes de parto doble.

En la (tabla II) se muestran los datos de calidad de canal. El peso de canal, característico de canales ligeras, no ha sido diferente entre los dos genotipos, ya que no hubo diferencias significativas en el rendimiento de la canal. Este último dato, en torno al $45 \%$ en ambos genotipos, es algo más bajo de lo esperado.

Los animales de padre Texel han tenido una mejor conformación $(\mathrm{p}=0,010)$ y un menor engrasamiento $(p \leq 0,001)$ que los de Segureña, algo esperable en una raza más cárnica y que conlleva un menor engrasamiento, resultados que coinciden con los encontrados por Vargas et al. (2015) en el cruce de Texel con la raza Pantaneiro Brasileña. Estos resultados, respecto a la conformación evaluada por medio de patrones fotográficos, fueron refrendados por la mayor compacidad de la pierna en los corderos cruzados de Texel, lo que da idea de su mejor morfología carnicera y, respecto al engrasamiento, por su menor porcentaje de grasa total y mayor porcentaje de músculo (4,5 puntos porcentuales mayor), en conformidad con lo encontrado por Valls (1980) en los cruces de Texel con Rasa Aragonesa. Estas diferencias en la grasa total se han debido al mayor desarrollo de la grasa preescapular y, sobre todo, subcutánea de los animales segureños, con 3,4 puntos porcentuales más que los animales cruzados, lo cual, dependiendo del circuito en el que se comercialice, es un dato a tener en cuenta en el caso de que un engrasamiento visual excesivo fuese penalizado por parte del mercado.

No obstante, los datos de calidad de canal de los corderos de la raza Segureña difieren de los observados por Cano et al. (2003) en conformación, porcentaje de músculo y grasa, lo que parece evidenciar una evolución de esta raza hacia un formato más compacto y magro.

\section{CONCLUSIONES}

El cruce industrial puede ser una herramienta útil en la producción de carne ovina y, en consecuencia, una vía para incrementar la rentabilidad de las explotaciones, al mejorar, en animales cruzados con Texel, los resultados productivos, en especial el peso al sacrificio, y obtenerse canales con una morfología más carnicera, con una significativa mayor cantidad de músculo y un menor porcentaje de grasa. No obstante, sería necesario analizar la calidad de la carne y su aceptabilidad por parte del consumidor y, por supuesto, la adaptación de estos animales al entorno y la rentabilidad del sistema.

\section{BIBLIOGRAFIA}

Cano, T.; Peña, F.; Martos, J.; Domenech, V.; Alcalde, M.J.; García, A.; Herrera, M.; Rodero, E. y Acero de la Cruz, R. 2003. Calidad de la canal y de la carne en corderos ligeros de raza Segureña. Arch Zootec, 52: 315-326.

Cardoso, M.T.M.; Landim, A.V.; Louvandini, H. and McManus, C. 2013. Performance and carcass quality in three genetic groups of sheep in Brazil. Rev Bras Zootecn, 42: 734-742.

Colomer, F.; Morand-Fehr, P.; Kirton, A.H.; Delfa, R. y Sierra, I. 1988. Mé-todos normalizados para el estudio de los caracteres cuantitativos y cualitativos de las canales caprinas y ovinas. Cuadernos INIA, 17: 41

Da Silva, A.F.; McManus, C.; Paim, T.P.; Dallago, B.S.L.; Esteves, G.I.F.; Louvandini, H.; Neto, J.B. y Lucci, C.M. 2014. Production traits in F1 and F2 crosses with naturalized hair breed Santa Inês ewes. SpringerPlus, 3: 66.

Espejo, M.; Mora, M. y García Barreto, L. 1977. Crecimiento y calidad 
carnicera de los productos obtenidos del cruce entre moruecos de razas cárnicas y ovejas de razas locales españolas. Anales INIA. Ser Prod Anim, 8: 55-67.

Esteban, C. 2003. Razas ganaderas españolas. II. Ovinas. Ed. MAPA. Madrid. España.

Kempster, A.J.; Croston, D. and Jones, D.W. 1987. Tissue growth and development in crossbred lambs sired by ten breeds. Liv Prod Sci, 16: 145-162.

Krener, R.; Barbato, G.; Castro, L.; Rista, L.; Rosés, L.; Herrera, V. and Nieirotti, V. 2004. Effects of sire breed, year sex and weight on carcass characteristics of lambs. Small Rum Res, 53: 117-124.

Mujica, F. (Ed.). 2005. Razas ovinas y caprinas en el Instituto de Investigaciones Agropecuarias. Osorno, Chile. Instituto de Investigaciones Agropecuarias. Bol INIA, 127: 88 .

Nitter, G. 1974. Results of a crossbredding experiment with sheep for intensive fat lamb production. Proceedings of the World Symposium of Breed Evaluation in Experimental Farm Animals, 376-387.

Osorio, J.C.; Da Costa, J.C.; Jardim, P.; Pouey, J.; Manzoni, N. e Osório, M.T. 1998. Morfologia e características comerciais da Produção de carne em cordeiros não castrados. 1. Efeito do genótipo. Anais da XXXV Reunião da SBZ. Botocatu. pp. 612-614.

Sañudo, C. 2011 . Atlas mundial de etnología zootécnica. Ed. Servet. Zaragoza. España. $818 \mathrm{pp}$

Sierra, I. 1981. Résultats du croisement industrile des beliers Fleiscschaf and Suffolk avec des brevis Rasa Aragonesa. Opt Mediterran, 81: 83-92.

Valls, M. 1980. Experiencia de cruce industrial. Memoria CRIDA 08, Producción Animal, 218.

Vargas, F.M.; Martins, C.F.; Pinto, G.S.; Ferreira, M.B.; Ricardo, H.A.; Leonardo, A.P.; Fernandes, A.R.M. and Teixeira, A. 2015. Carcass measurements, non carcass components and cut production of local Brazilian Pantaneiro sheep and crossbreeds of Texel and Santa Inês with Pantaneiro. Small Rum Res, DOI:10.1016/j.smallrumres. 2014.12.007

Vergara, H. 2005. Composición regional y tisular de la canal ovina. En: Cañeque, V. y Sañudo, C. (Coor.). Estandarización de las metodologías para evaluar la calidad del producto en los rumiantes. INIA. Madrid. España. Monografías INIA, 3: 170-178. 